(2) Open Access Full Text Article

ORIGINAL RESEARCH

\title{
Association of patient-rated severity with other outcomes in patients with painful diabetic peripheral neuropathy
}

This article was published in the following Dove Press journal:

Diabetes, Metabolic Syndrome and Obesity:Targets and Therapy 2 December 2011

Number of times this article has been viewed

\author{
Gavin Taylor-Stokes' \\ James Pike' \\ Alesia Sadosky² \\ Arthi Chandran ${ }^{2}$ \\ Thomas Toelle ${ }^{3}$
}

'Adelphi Real World, Adelphi Mill, Bollington, Macclesfield, Cheshire, UK; ${ }^{2}$ Pfizer Inc, New York, NY, USA; ${ }^{3}$ Department of Neurology, Technische Universität München, Munich, Germany
Correspondence: Alesia Sadosky Pfizer Inc, 235 East 42nd Street, New York, NY 10017, USA

Tel + I $212733949 \mid$

Fax + I $64644 \mid 4757$

Email alesia.sadosky@pfizer.com
Objective: To evaluate the association of patient-reported severity of painful diabetic peripheral neuropathy ( $\mathrm{pDPN}$ ) with other outcomes in a European population of patients using the Adelphi Disease Specific Programme for pDPN (DSP III, 2008).

Methods: The severity of patients' pDPN (mild, moderate, or severe) was rated independently by both patients and physicians. Relationships were evaluated between patient-reported pDPN severity and other patient-reported outcomes including pain, sleep, function, and work productivity. Physicians rated the severity of patients' $\mathrm{pDPN}(1=$ mild, $2=$ moderate, $3=$ severe $)$ and sleep interference.

Results: Patient-reported data were available from 634 individuals (56.2\% male, mean age 63 years) from France, Germany, Italy, and the UK, of whom only $22.2 \%$ reported that they were currently employed. pDPN severity was rated as mild, moderate, and severe by $22.2 \%$, $60.9 \%$, and $16.9 \%$ of the patients, respectively. There was a significant association between patient-rated and physician-rated pDPN severity $(P<0.0001)$, although there were discrepancies in agreement $(\mathrm{kappa}=0.37,95 \%$ confidence interval $[\mathrm{CI}] 0.31,0.43$; weighted kappa $=0.43$, $95 \%$ CI $0.37,0.48$ ) among physician and patient ratings in a substantial proportion of patients across severity categories. Higher pDPN severity was associated with greater interference of daily function including sleep ( $P<0.0001$ for all pairwise comparisons). Among employed patients, percent of pDPN-related impairment while at work (presenteeism) and overall work impairment increased with greater pDPN severity, resulting in indirect costs that increased significantly with pDPN severity; $\$ 8266, \$ 15,449$, and $\$ 24,300$ for mild, moderate, and severe pDPN, respectively (overall $P<0.001$ ).

Conclusion: Severity of patient-rated pDPN was significantly associated with outcomes, including function and productivity; poorer function and lower productivity were reported at higher $\mathrm{pDPN}$ severity levels. Moreover, physicians rated $\mathrm{pDPN}$ severity different from patients in a substantial proportion of patients.

Keywords: diabetic neuropathy, pain, outcomes

\section{Introduction}

The diabetic neuropathies comprise a family of nerve disorders that are common complications of diabetes and can result in chronic, persistent, neuropathic pain. ${ }^{1}$ These neuropathies occur with a similar frequency among patients with type 1 and type 2 diabetes, 59\% and $66 \%$, respectively. ${ }^{2}$ Painful diabetic peripheral neuropathy (pDPN) is a variant of distal symmetrical sensorimotor polyneuropathy, the most common form of diabetic neuropathy. ${ }^{3}$ Although best estimates suggest that only $15 \%$ of patients with diabetes have $\mathrm{pDPN},{ }^{1}$ the symptoms of chronic pain and reduced 
function have a profound impact on patients and result in a substantial burden relative to both the general population and to diabetic patients without pDPN. ${ }^{1,4-14}$

Although updated guidelines for the treatment of neuropathic pain have recently been published, ${ }^{15-18}$ treatment of pDPN remains challenging, especially given the presence of comorbidities, contraindications, and use of other medications with potential interactions. ${ }^{19}$

Understanding pDPN severity and its impact on the patient and societal burdens can help inform development and application of appropriate management strategies. In particular, given the subjective and multidimensional nature of pain, such an understanding is especially important when considered from the patients' perspective.

The identification of established cut points for characterizing mild, moderate, and severe pain associated with $\mathrm{pDPN}^{20}$ has enhanced the ability to evaluate the relationship between pain and other outcomes. Several studies have evaluated these relationships, and reported that the magnitude of the effects of pDPN pain on outcomes of function, sleep, quality of life, depression, and anxiety was higher at greater levels of pain severity. ${ }^{9-11,21}$ However, a broader approach that considers severity of pDPN as a condition, not just pain severity, may provide a more clinically relevant patient perspective of severity and impact. Such an approach has been used in other painful conditions including osteoarthritis and chronic low back pain. ${ }^{22-24}$ Additionally, only limited quantitative data exist on the impact of pDPN on productivity. One study reported that lost productive time among diabetic patients with neuropathic symptoms was higher than among diabetic patients without such symptoms, and $18 \%$ higher than nondiabetic controls. ${ }^{6}$ A more recent study demonstrated that work productivity was significantly lower with increasing pain severity, and that costs related to lost productivity increased with greater levels of pain. ${ }^{21}$ Therefore, the purpose of this study was to explore the relationship between patientreported severity of $\mathrm{pDPN}$ and health indicators, as well as to quantitatively assess the impact of pDPN severity on productivity.

\section{Methods}

\section{Data source}

The Adelphi Disease Specific Program (DSP) represents an aggregated database from a cross sectional study of consulting patients, which includes patient-level and physician-reported data on specific chronic diseases. ${ }^{25}$ Data are collected in clinical practice settings by physicians who provide relevant information on consecutive patients who consulted for the disease of interest. Patients are invited to participate by completing questionnaires on symptoms, expectations, and QOL. The current analysis, which is based on the Neuropathic Pain III DSP conducted in 2008 in Europe among patients with a variety of neuropathic pain conditions, focuses on the subset of patients who were identified with a confirmed diagnosis of pDPN. Data are included from subjects from France, Germany, Italy, and the United Kingdom. Physician practices captured by this DSP were those in primary care as well as the following specialties: neurology, anesthesiology, diabetology, orthopedic surgery, and rheumatology.

\section{Outcomes}

In contrast to other studies that used a single question asking patients to rate their disease severity as mild, moderate, or severe, this analysis did not rely on a direct question, but rather determined pDPN severity based on two questions. The first question asked patients to identify and rate (mild, moderate, and severe) their most common types of neuropathic pain sensations (hot/burning, tingling, aching, shooting, stabbing, sharp, pins and needles, numbness, tight, electrical, dull, other). The second question asked patients to identify painful areas of the body and rate the pain of their most commonly painful body part. While patients could identify multiple body locations of pain, only a single body part was considered for pain severity. When considering the overall rating of pDPN severity, the default was severity of neuropathic pain sensations if there was discordance between the two ratings.

Patient-reported pain severity and interference with function was captured using the Brief Pain Inventory-Short Form (BPI-SF). ${ }^{26}$ Four questions focus on pain severity using a $0-10$ numerical rating scale $(\mathrm{NRS} ; 0=$ no pain, $10=$ pain as bad as you can imagine): worst, least, average, and current pain with a 24-hour recall period for the first three items. Patients were stratified as having mild, moderate, or severe pain using the cut points of $0-3,4-6$, and 7-10, respectively, which were previously identified in patients with pDPN. ${ }^{20}$ Seven items on the BPI-SF measure pain interference with daily function $(0=$ does not interfere, $10=$ completely interferes $)$, including sleep. Pain severity and pain interference with daily function were assessed by averaging the four pain severity items and the seven pain interference items, respectively. Additionally, evaluation of pDPN severity on sleep was specifically evaluated using the appropriate item from the BPI-SF for patient-reported sleep, and physician's assessment based on the question "In your opinion to what extent does the patient's neuropathic pain interfere with sleep?" 
Work productivity was captured using the Work Productivity and Activity Impairment scale (WPAI). ${ }^{27}$ The WPAI consists of six questions with the first question on employment status. The remaining five questions, referenced to the past 7 days, relate to hours missed because of neuropathic pain; hours missed because of other reasons; hours actually worked; degree neuropathic pain affected productivity while working (rating scale from $0=$ no effect to $10=$ completely prevented from working); and degree neuropathic pain affected regular activities (rating scale $0=$ no effect to $10=$ completely prevented daily activities). By summing and dividing these responses accordingly, the percent work time missed due to pDPN (absenteeism) can be calculated, as well as the percent impairment while on the job due to this condition (presenteeism), percent overall work impairment and the percent activity impairment due to their condition. Costs, in euros and dollars, due to lost productivity were calculated using the method of Lofland et $\mathrm{al}_{,}{ }^{28}$ based on average annual wages for 2008 in the EU estimated from published sources (http://data.worldbank. org/indicator/NY.GNP.PCAP.CD) and the average dollar to euro rate for 2008 (http://www.irs.gov/businesses/small/ international $/$ article $/ 0$, id $=206089,00 . \mathrm{html})$.

Physicians also provided a rating of patient's pDPN severity in response to the question "Which one of these best describes your view of the severity of the patient's neuropathic pain condition?" with potential responses of $1=$ mild, $2=$ moderate, $3=$ severe, $4=$ very severe. Physician ratings were obtained independently of the patient's ratings.

\section{Statistical analysis}

The relationship between severity of neuropathic pain sensations and severity of the most commonly painful body part was specifically evaluated using the nonparametric Spearman rank correlation. Overall patient-reported pDPN severity, stratified by mild, moderate, and severe, was further evaluated for relationships with other patient-reported outcomes. Pre-specified analyses were performed using STATA 10.1 data analysis and statistical software (StataCorp LP, College Station, Texas, USA). Analysis of variance (ANOVA) and $t$-tests were used for interval and count data; chi-squared tests, or Fisher exact test for binary variables were used for categorical data; and Kruskal-Wallis tests or Mann-Whitney tests were conducted on pairwise severity comparisons for ordinal data. All multiple comparisons were Bonferroni corrected. Evidence for statistical significance was based on a $P$ value $<0.05$.

\section{Results}

Patient-reported data were available from 634 individuals identified as having a confirmed diagnosis of pDPN, $162(25.6 \%)$ from France, 230 (36.3\%) from Germany, $158(24.9 \%)$ from Italy, and $84(13.2 \%)$ from the UK. Diagnosis was confirmed by a diabetologist or neurologist in $30.1 \%$ and $28.3 \%$ of cases, respectively, followed by primary care physicians $(24.8 \%)$; the remaining $16.8 \%$ of cases were confirmed by other physician specialties or a combination of physician types. The demographic characteristics of this population, shown in Table 1 indicate that they were predominantly male $(56.2 \%)$, with a mean age of $63.0 \pm 11.4$ years, and a mean time since pDPN diagnosis of $1.8 \pm 2.1$ years; approximately half the population $(50.4 \%)$

Table I Demographic characteristics of the study population $(\mathrm{N}=634)$

\begin{tabular}{|c|c|}
\hline Characteristic & Value \\
\hline \multicolumn{2}{|l|}{ Gender, n (\%) } \\
\hline Male & $355(56.2)$ \\
\hline Female & $277(43.8)$ \\
\hline \multicolumn{2}{|l|}{ Age, years } \\
\hline Mean \pm standard deviation & $63.0 \pm 11.4$ \\
\hline Range & $23-89$ \\
\hline \multicolumn{2}{|l|}{ Country, n (\%) } \\
\hline France & $162(25.6)$ \\
\hline Germany & $230(36.3)$ \\
\hline Italy & $158(24.9)$ \\
\hline United Kingdom & $84(13.3)$ \\
\hline \multicolumn{2}{|l|}{ Ethnicity, n (\%) } \\
\hline White & $595(94.2)$ \\
\hline Non-white & $37(5.9)$ \\
\hline \multicolumn{2}{|l|}{ Employment status, n (\%) } \\
\hline Full-time & $128(20.5)$ \\
\hline Part-time & II (I.8) \\
\hline Homemaker & $59(9.4)$ \\
\hline Retired & $365(58.3)$ \\
\hline Unemployed & $63(10.1)$ \\
\hline \multicolumn{2}{|l|}{ Body mass index } \\
\hline Mean \pm standard deviation, $\mathrm{kg} / \mathrm{m}^{2}$ & $27.2 \pm 4.8$ \\
\hline$\geq 30, \mathrm{n}(\%)$ & $318(50.4)$ \\
\hline \multicolumn{2}{|l|}{ Time since diagnosis, years } \\
\hline Mean standard deviation & $1.8 \pm 2.1$ \\
\hline Range & $0-17.3$ \\
\hline \multicolumn{2}{|c|}{ Common comorbidities ( $\geq 5 \%$ of patients), $n(\%)$} \\
\hline Hypertension & $369(58.2)$ \\
\hline Hypercholesterolemia & $246(38.8)$ \\
\hline Cardiovascular conditions & $110(17.4)$ \\
\hline Sleep disorders & $107(16.9)$ \\
\hline Osteoarthritis & $95(15.0)$ \\
\hline Clinical depression & $86(13.6)$ \\
\hline Feelings of depression & $66(10.4)$ \\
\hline Clinical anxiety & $48(7.8)$ \\
\hline Feelings of anxiety & $44(6.9)$ \\
\hline
\end{tabular}

Note: Numbers may not add up to 634 because of missing data. 
had a body mass index $\geq 30$, indicating obesity. Only $22.4 \%$ of subjects reported that they were currently employed; $58.3 \%$ were retired, $10.1 \%$ were unemployed, and $9.4 \%$ stated that they were homemakers.

Pain was reported in multiple body sites in $38.5 \%$ of patients. Overall, patients identified the lower extremities (leg or foot) as their most frequent location of neuropathic pain (91.4\%). Among patients who reported a single body location for pain $(n=375), 92.8 \%$ reported neuropathic pain in the leg or foot.

The proportion of patients who rated their pDPN condition as mild, moderate, and severe was $22.2 \%, 60.9 \%$, and $16.9 \%$, respectively, and these proportions were similar across the countries (Figure 1). The two items used to assess condition severity were strongly correlated (Spearman $r=0.7165)$, with substantial agreement between ratings also indicated by the kappa coefficient $(0.6087,95 \%$ CI 0.5504 , $0.6670)$ and the weighted kappa $(0.6516,95 \%$ CI 0.5940 , 0.7091). There was no association of pDPN severity with demographic variables including age, gender, BMI, or overall employment status. Similarly, there was no association of pDPN severity with disease duration.

For physician-rated severity, since there were only 8 patients who were rated as very severe, these patients were incorporated into the severe category. There was a clear and significant association observed between patient-rated and physician-rated severity $(P<0.0001)$. However, when analyzed using a 3-by-3 contingency table (Table 2), ratings showed discrepancies in agreement $(\mathrm{kappa}=0.37,95 \%$ CI 0.31, 0.43; weighted kappa $=0.43$, 95\% CI $0.37,0.48)$. Discordance between physician and patient ratings was evident in a substantial proportion of patients across severity categories, and was especially

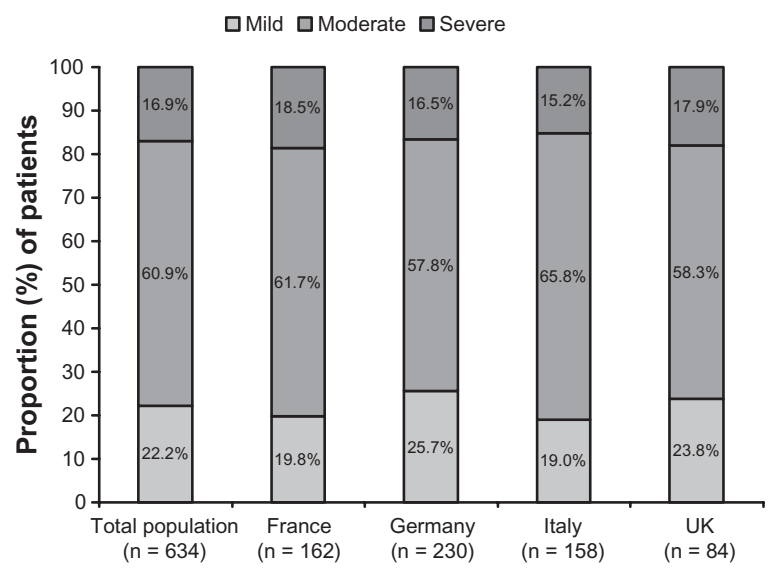

Figure I Proportion of patients rating their painful diabetic peripheral neuropathy as mild, moderate, and severe. noticeable with physicians underestimating $46.7 \%$ of the patients who reported severe $\mathrm{pDPN}$.

Pain severity and pain interference with daily function demonstrated significant increases with increasing pDPN severity (Figure 2). Impact on sleep also significantly increased with increasing pDPN severity $(P<0.0001)$ (Figure 3), and all pairwise severity comparisons for both patient and physician assessments of sleep were significant $(P<0.0001)$.

Among the employed individuals for whom WPAI data were available, there was a significant association between pDPN severity and work productivity $(P<0.05)$ (Figure 4$)$. In particular, the percent overall activity impairment was significantly higher with increasing pDPN severity levels ( $P<0.0001$ for all pairwise comparisons). Employed individuals reported greater impairment while working and greater overall working impairment at increasing pDPN severity levels, which were significant for mild versus severe $(P<0.001)$ and moderate versus severe $(P<0.05)$. Estimated annual costs related to lost work productivity among employed individuals with pDPN increased significantly with pDPN severity (Figure 5); €5,646 (\$8,266), €10,552 (\$15,449), and $€ 16,597$ (\$24,300) for mild, moderate, and severe pDPN, respectively (overall $P=0.0002$ and $P<0.05$ for all pairwise comparisons). These costs appeared to be primarily driven by presenteeism, ie, impairment while working. Mean total costs of lost productivity across severity categories were $€ 15,446 \pm 12,272(\$ 10,549 \pm 8,383)$ and were similar among the countries; $€ 8,185 \pm 6,376(\$ 11,984 \pm 9,335)$ for Italy, $€ 10,789 \pm 8,823(\$ 15,797 \pm 12,918)$ for France, $€ 10,828 \pm 9,042(\$ 15,853 \pm 13,238)$ for Germany, and $€ 12,438 \pm 8,573(\$ 18,211 \pm 12,552)$ for the UK.

\section{Discussion}

An understanding of the relationship between pDPN severity and other outcomes affected by this condition can help characterize the disease burden and enhance development and application of appropriate management strategies. The results presented here, which represent a subset of patients with pDPN from a survey of patients with neuropathic pain conditions, provide a European perspective and expand on previous studies that showed a significantly greater patient burden associated with pDPN at higher pain severity levels. ${ }^{9,11}$ In contrast to those studies, which specifically evaluated pain severity, the current analysis included more than just pain to define pDPN as a condition, with particular emphasis on neuropathic pain-related symptoms. Importantly, the strong correlation between severity of sensations and body part 
Table 2 Physician-reported versus patient-reported severity of painful diabetic neuropathy as a condition (weighted kappa coefficient $=0.43$; $95 \%$ confidence interval $0.37,0.48$ )

\begin{tabular}{|c|c|c|c|c|c|c|}
\hline \multirow{2}{*}{$\begin{array}{l}\text { Patient-reported } \\
\text { severity }\end{array}$} & & \multicolumn{3}{|c|}{ Physician-reported severity, n } & \multirow{2}{*}{$\begin{array}{l}\text { Row total, } \\
\text { n (\%) }\end{array}$} & \multirow{2}{*}{$\begin{array}{l}\text { pDPN severity discordance, } \\
\text { percent of patients }\end{array}$} \\
\hline & & Mild & Moderate & Severe* & & \\
\hline Mild & Frequency & 80 & 52 & 6 & $138(22.0)$ & 42.0 \\
\hline Moderate & Frequency & 45 & 268 & 70 & $383(61.2)$ & 30.0 \\
\hline Severe & Frequency & 3 & 46 & 56 & $105(16.8)$ & 46.7 \\
\hline Column total, n (\%) & & $128(20.5)$ & $366(58.5)$ & I $32(2 \mid .1)$ & $626(100)$ & 35.5 \\
\hline
\end{tabular}

Note: *Includes "very severe" ( $\mathrm{n}=8)$.

Abbreviation: pDPN, painful diabetic peripheral neuropathy.

severity suggests that pain is not the sole component driving the rating of pDPN severity. Additionally, these results show that pDPN severity correlated well with pain, sleep, interference with function, and work productivity.

Information on body regions affected by pDPN is lacking. One study, in a small number of patients, reported that neuropathic pain was always located in the feet, ${ }^{29}$ and evidence from other studies suggested that trigeminal and facial nerves may also be affected. ${ }^{30,31}$ While the lower extremities were the most commonly reported source of neuropathic pain in the current study, the anatomic location of neuropathic pain was not limited to this region of the body, and approximately half the patients reported neuropathic pain in other body parts, although quantitative sensory testing or neurophysiology to confirm the presence of neuropathic pain was not performed.

As pDPN severity increased, there was a corresponding increase in pain severity, pain interference with daily function, sleep, employment status, and work-related productivity impairment. Notably, with increasing pDPN severity, the change in pain severity was matched by the change in pain interference with function, suggesting close association between the patient's perception of pDPN with that of both pain and functional ability. Pain interference at

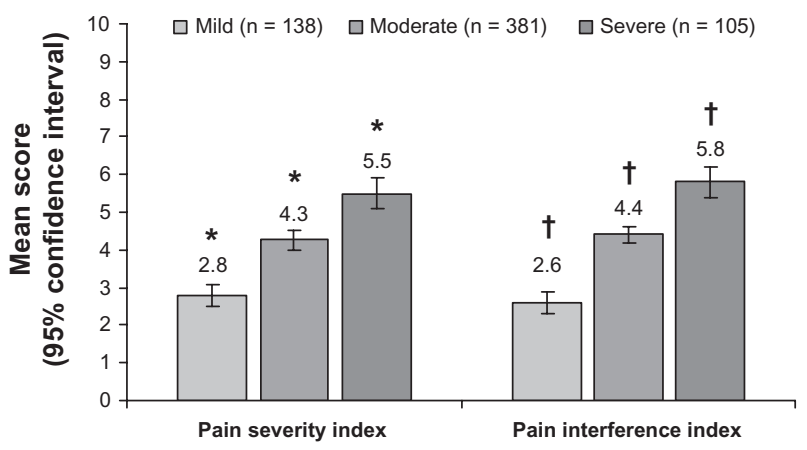

Figure 2 Relationship between self-reported severity of painful diabetic peripheral neuropathy and the Brief Pain Inventory Severity Indices.

Notes: $*, p<0.000$ I for all pairwise comparisons between severity levels. each pDPN severity level in the current study was within the range of values reported in previous studies for increasing pain severity levels. ${ }^{9,11}$

Especially notable was the significant association between pDPN severity and pain-related sleep interference, which was consistent with a previous study that showed greater sleep impairment at higher levels of pain severity. ${ }^{9}$ These associations are of clinical relevance given that pDPN not only results in substantial sleep impairment, ${ }^{32}$ but that sleep impairment has been shown to have a direct relationship with metabolic control, including increased risk for and severity of diabetes. ${ }^{33}$

Among patients who remained in the workforce, severity of pDPN was associated with lost productivity. Interestingly, previous research has shown that among employed individuals with diabetes, those having neuropathic symptoms have significantly greater work loss than those without these symptoms. ${ }^{6}$

Our data suggest that among employed individuals, the magnitude of work loss increases with pDPN severity, and that most of this lost productivity is derived from impairment while at work, ie, presenteeism. Patients with moderate severity reported that approximately one-third of their work time (36.8\%) was lost due to pDPN, and almost twice as

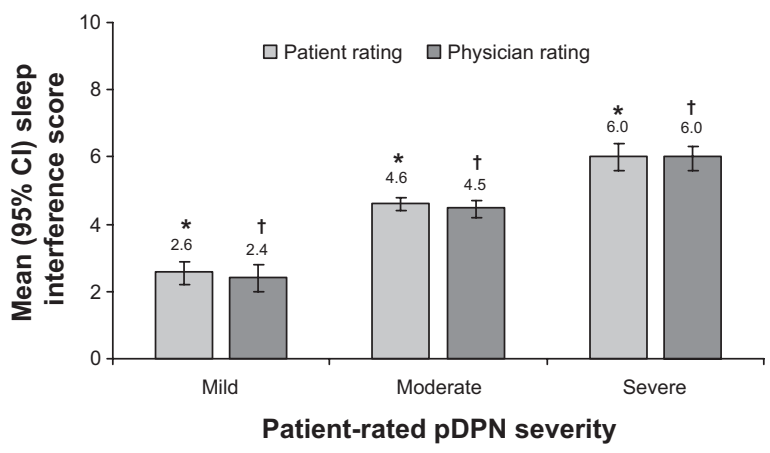

Figure 3 Relationship between self-reported severity of painful diabetic peripheral neuropathy and impact of pain on sleep.

Notes: $P<0.0001$ for overall effect within patient and physician ratings; $*,+P<0.000$ I for all pairwise comparisons between severity levels.

Abbreviations: $\mathrm{Cl}$, confidence interval; pDPN, painful diabetic peripheral neuropathy. 


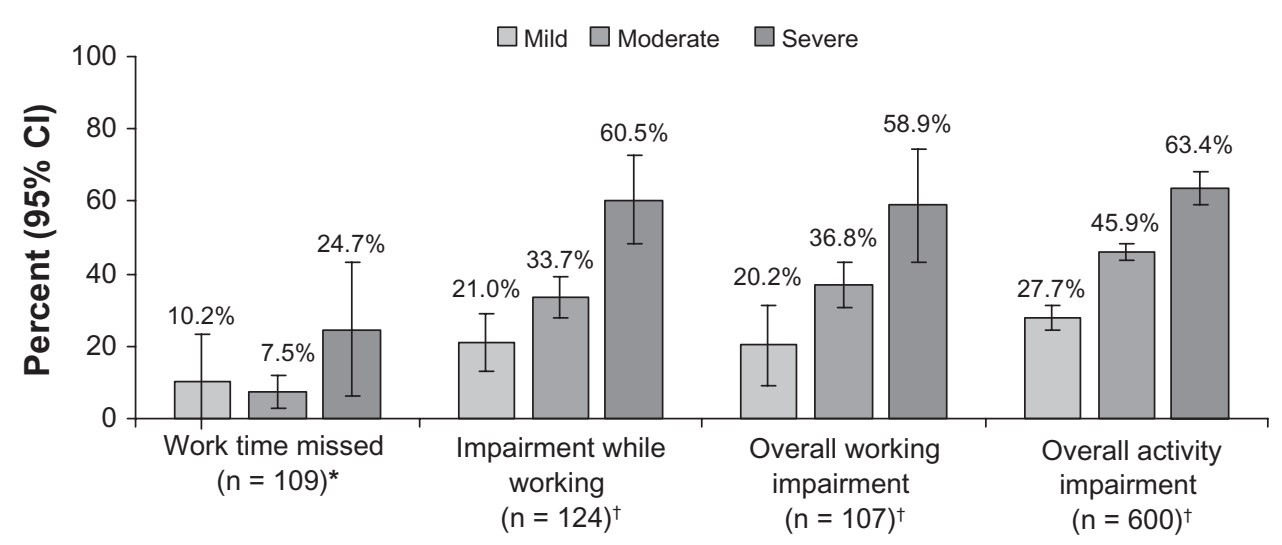

Work productivity and impairment scale

Figure 4 Relationship between self-reported severity of painful diabetic peripheral neuropathy and productivity assessed using the Work Productivity and Activity Impairment (WPAI) scale. ${ }^{27}$

Notes: $* P<0.05 ;{ }^{\dagger} P<0.00$ I for overall effect.

Abbreviation: $\mathrm{Cl}$, confidence interval.

much lost work time (58.9\%) was lost by patients with severe $\mathrm{pDPN}$. These trends were similar to those observed in a US study that reported on pain severity and productivity in patients with pDPN. ${ }^{21}$ Additionally, these observations are consistent with the suggestion that presenteeism is the primary source of lost productive time, and is also likely to be the primary driver of indirect costs among workers with chronic pain conditions. ${ }^{34}$ In fact, indirect costs associated with lost productivity in the current study were significantly associated with pDPN severity, with indirect costs for a patient with severe pDPN $(€ 16,597)$ approximately three times that of a patient with mild pDPN $(€ 5,646)$.

Although there was some agreement between patient- and physician-rated pDPN severity (weighted kappa $=0.43$ ), there was a substantial disparity between the physicians' and patients' ratings, with approximately one-third of patients' ratings being over- or under-rated by physicians, including almost half of the patients who rated themselves as severe being rated by the physicians as mild or moderate. This result is consistent with the general finding in other conditions that clinicians and patients have different perspectives of severity, both underestimating and overestimating specific outcomes or overall disease severity. ${ }^{23,35-41}$ Such an effect, where physicians underestimate the severity of a patient's condition, can conceivably result in a proportion of patients not receiving appropriate treatment. Conversely, if overestimated, the patients may receive medications that are not needed, unnecessarily increasing the risks that may be associated with specific medications.

Although the questions used to determine patient-rated severity were specific for neuropathic pain, it is possible that other factors contributed to these ratings and may in part account for the discordance between patient and physician ratings. In particular, the presence of pain-related comorbidities as well as neuropsychiatric conditions such as depression and anxiety, which were present in this population,

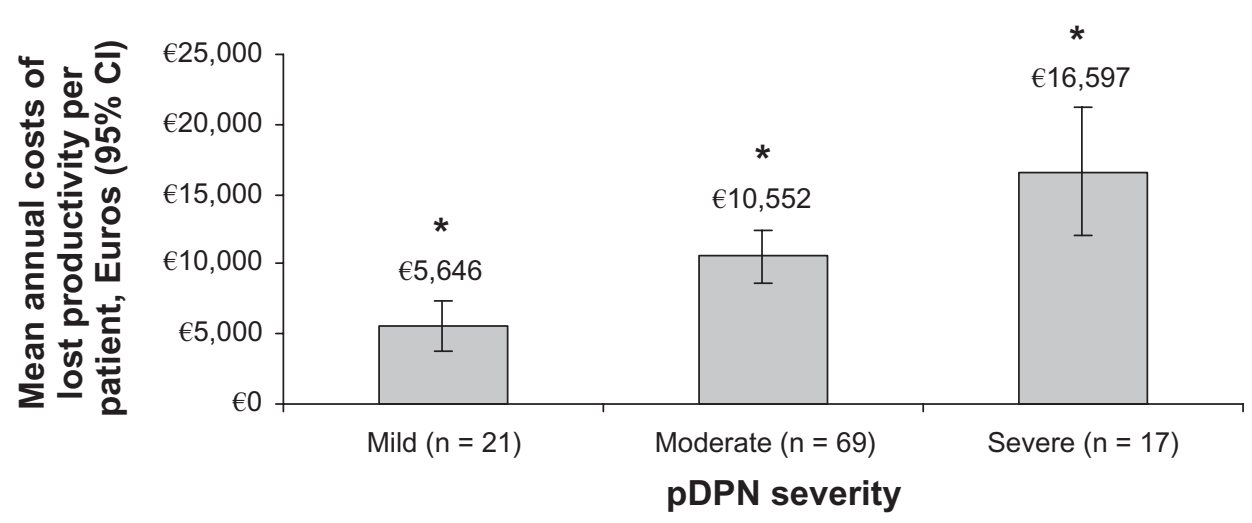

Figure 5 Relationship between self-reported severity of painful diabetic peripheral neuropathy and indirect costs resulting from lost productivity. Notes: ${ }^{*} P<0.05$ for all pairwise comparisons between severity levels; $P=0.002$ for overall effect. Abbreviation: pDPN, painful diabetic peripheral neuropathy. 
could potentially have an influence on patient perceptions of pDPN. Nevertheless, this disparity has several clinical implications, including the need for identifying factors contributing to severity that may be emphasized differentially by patients and physicians. This discrepancy strongly argues for the importance of the patient's perspective, and suggests that physicians need to work closely with patients to gain a comprehensive assessment.

Interpretation and extrapolation of these results are subject to several limitations, including the fact that participation was based on agreement by physicians and patients. It is therefore possible that individuals who participated may have characteristics and perceptions different from those who refused to participate, thereby introducing selection bias and reducing generalizability.

While the population was drawn from a sample of patients with neuropathic pain, the demographics suggest that these patients are likely to be representative of patients with pDPN in the general diabetes population; older age, predominantly male, and with a presence of risk factors for diabetes including obesity, hypertension, and hypercholesterolemia.

Recall bias when completing a questionnaire is another potential limitation, which was minimized for all of the measures by using a maximum recall period of the past 7 days. Furthermore, because the data are only from 4 countries they may not necessarily be representative of the European population, indicating the need for broader sampling.

That the type of employment was not captured in the questionnaire may also be considered a limitation, because it is likely to affect the magnitude of productivity loss among workers as well as the likelihood that employment status was compromised among those who stopped work because of their pDPN. Similarly, the analyses were not adjusted for the specific body parts where patients reported their most commonly occurring pain, because the body site is likely to affect daily function and productivity.

With regard to the diagnosis of pDPN for inclusion, this diagnosis is dependent on the diagnostic skill of the treating physician. Although diabetologists and neurologists were the diagnosing physicians in more than half the patients, the basis for the diagnosis (ie, quantitative sensory testing, neurography, etc) was not captured as part of the study, and therefore it is possible that misdiagnosis may have occurred in a small proportion of the sample population.

It should be noted that the cross-sectional nature of DSPs precludes causation. Since no cause and effect imputation can be made, the observed relationships should be considered associative rather than causal.

\section{Conclusion}

Severity of pDPN was significantly associated with pain, sleep, function, and lost productivity; poorer function and sleep, and lower productivity were reported at higher pDPN severity levels, with lower productivity also having a greater economic impact. In a proportion of patients there was substantial discordance between physician and patient ratings of pDPN severity. Further analyses of these observed relationships are warranted from the perspectives of both the patients and clinicians with the goal of refining targeted management strategies to meet the needs of the patients.

\section{Disclosure}

This study was funded by Pfizer Inc. Messers TaylorStokes and Pike are full-time employees of Adelphi, who were paid consultants to Pfizer in connection with the analysis and development of this manuscript. Dr Sadosky and Ms Chandran are employees of Pfizer Inc. Dr Toelle was involved in all aspects of the project, but was not financially compensated for his collaboration. Editorial support was provided by E Jay Bienen, who was funded by Pfizer Inc.

\section{References}

1. Sadosky A, McDermott AM, Brandenburg NA, Strauss M. A review of the epidemiology of painful diabetic peripheral neuropathy, postherpetic neuralgia, and less commonly studied neuropathic pain conditions. Pain Pract. 2008;8(1):45-56.

2. Dyck PJ, Kratz KM, Karnes JL, et al. The prevalence by staged severity of various types of diabetic neuropathy, retinopathy, and nephropathy in a population-based cohort: the Rochester Diabetic Neuropathy Study. Neurology. 1993;43(4):817-824.

3. Boulton AJM, Vinik AI, Arezzo JC, et al. Diabetic neuropathies. A statement by the American Diabetes Association. Diabetes Care. 2005; 28(4):956-962.

4. Benbow SJ, Wallymahmed ME, Macfarlane IA. Diabetic peripheral neuropathy and quality of life. QJ Med. 1998;91:733-737.

5. Daousi C, MacFarlane IA, Woodward A, Nurmikko TJ, Bundred PE, Benbow SJ. Chronic painful peripheral neuropathy in an urban community: a controlled comparison of people with and without diabetes. Diabet Med. 2004;21(9):976-982.

6. Stewart WF, Ricci JA, Chee E, Hirsch AG, Brandenburg N. Lost productive time and costs due to diabetes and diabetic neuropathic pain in the US workforce. J Occup Environ Med. 2007;49(6):672-679.

7. Galer BS, Gianas A, Jensen MP. Painful diabetic polyneuropathy: epidemiology, pain description, and quality of life. Diabetes Res Clin Pract. 2000;47(2):123-128.

8. Gore M, Brandenburg N, Hoffman D, Tai K-S, Stacey B. Burden of illness in painful diabetic peripheral neuropathy (DPN): the patients' perspective. J Pain. 2006;7(12):892-900.

9. Gore M, Brandenburg N, Dukes E, Hoffman D, Tai K-S, Stacey B. Pain severity in diabetic peripheral neuropathy is associated with patient functioning, symptom levels of anxiety and depression, and sleep. J Pain Symptom Manage. 2005;30(4):374-385.

10. Hoffman DL, Sadosky A, Alvir J. Cross-national burden of painful diabetic peripheral neuropathy in Asia, Latin America, and the Middle East. Pain Pract. 2009;9(1):35-42. 
11. Tölle T, Xu X, Sadosky AB. Painful diabetic neuropathy: a crosssectional survey of health state impairment and treatment patterns. J Diabetes Complications. 2006;20(1):26-33.

12. Ritzwoller DP, Ellis JL, Korner EJ, Hartsfield CL, Sadosky A. Comorbidities, healthcare service utilization and costs for patients identified with painful DPN in a managed-care setting. Curr Med Res Opin. 2009;25(6):1319-1328.

13. Dworkin RH, Malone DC, Panarites CJ, Armstrong EP, Pham SV. Impact of postherpetic neuralgia and painful diabetic peripheral neuropathy on health care costs. J Pain. 2010;11(4):360-368.

14. Dworkin RH, Panarites CJ, Armstrong EP, Malone DC, Pham SV. Healthcare utilization in people with postherpetic neuralgia and painful diabetic peripheral neuropathy. J Am Geriatr Soc. 2011;59(5): $827-836$.

15. Attal N, Cruccu G, Baron R, et al. EFNS guidelines on the pharmacological treatment of neuropathic pain: 2010 revision. Eur J Neurol. 2010;17(9):e1113-e1188.

16. Dworkin RH, O'Connor AB, Audette J, et al. Recommendations for the pharmacological management of neuropathic pain: an overview and literature update. Mayo Clin Proc. 2010;85(Suppl 3):S3-S14.

17. Centre for Clinical Practice. Neuropathic pain. The pharmacological management of neuropathic pain in adults in non-specialist settings. London: National Institute for Health and Clinical Excellence (NICE); 2010 Mar. 155p. (Clinical guideline; no. 96).

18. Bril V, England J, Franklin GM, et al. Evidence-based guideline: Treatment of painful diabetic neuropathy: Report of the American Academy of Neurology, the American Association of Neuromuscular and Electrodiagnostic Medicine, and the American Academy of Physical Medicine and Rehabilitation. Neurology. 2011;76(20): $1758-1765$.

19. Gore M, Sadosky A, Leslie D, Sheehan AH. Selecting an appropriate medication for treating neuropathic pain in patients with diabetes: a study using the UK and Germany Mediplus databases. Pain Pract. 2008;8(4):253-262.

20. Zelman D, Dukes E, Brandenburg N, Bostrom A, Gore M. Identification of cut-points for mild, moderate and severe pain due to diabetic peripheral neuropathy. Pain. 2005;115(2):29-36.

21. Dibonaventura MD, Cappelleri JC, Joshi AV. Association between pain severity and health care resource use, health status, productivity and related costs in painful diabetic peripheral neuropathy patients. Pain Med. 2011;12(5):799-807.

22. Sadosky A, Bushmakin A, Cappelleri JC, Lionberger DR. Relationship between patient-reported disease severity in osteoarthritis and self-reported pain, function, and work productivity. Arthritis Res Ther. 2010;12:R162.

23. Bushmakin AG, Cappelleri JC, Taylor-Stokes G, et al. Relationship between patient-reported disease severity and other clinical outcomes in osteoarthritis: a European perspective. J Med Econ. 2011;14(4): 381-389.

24. Sadosky A, Taylor-Stokes G, Lobosco S, Pike J, Ross E. Relationship between self-reported low back pain severity and other patient-reported outcomes: results from an observational study. J Spinal Disord Tech. 2011. In press
25. Anderson P, Benford M, Harris N, Karavali M, Piercy J. Real-world physician and patient behaviour across countries: disease-specific programmes - a means to understand. Curr Med Res Opin. 2008;24(11): 3063-3072.

26. Cleeland CS. Pain assessment in cancer. In: Osoba D, editor. Effect of Cancer on Quality of Life. Boca Raton, FL: CRC Press; 1991:293-305.

27. Reilly MCZ, AS, Dukes EM. The validity and reproducibility of a work productivity and activity impairment instrument. Pharmacoeconomics. 1993;4(5):353-365.

28. Lofland JH, Pizzi L, Frick KD. A review of health-related workplace productivity loss instruments. Pharmacoeconomics. 2004;22(3): 165-184.

29. Vrethem M, Boivie J, Arnqvist H, Holmgren H, Lindstrom T. Painful polyneuropathy in patients with and without diabetes: clinical, neurophysiologic, and quantitative sensory characteristics. Clin J Pain. 2002; 18(2):122-127.

30. Urban PP, Forst T, Lenfers M, Koehler J, Connemann BJ, Beyer J. Incidence of subclinical trigeminal and facial nerve involvement in diabetes mellitus. Electromyogr Clin Neurophysiol. 1999;39(5):267-272.

31. Irkec C, Nazliel B, Yetkin I, Kocer B. Facial nerve conduction in diabetic neuropathy. Acta Neurol Belg. 2001;101(3):177-179.

32. Zelman D, Brandenburg NA, Gore M. Sleep impairment in patients with painful diabetic peripheral neuropathy. Clin J Pain. 2006;22(8): 681-685.

33. Tasali E, Leproult R, Spiegel K. Reduced sleep duration or quality: relationships with insulin resistance and type 2 diabetes. Prog Cardiovasc Dis. 2009;51(5):381-391.

34. Stewart WF, Ricci JA, Chee E, Morganstein D, Lipton R. Lost productive time and cost due to common pain conditions in the US workforce. JAMA. 2003;290(18):2443-2454.

35. Suarez-Almazor ME, Conner-Spady B, Kendall CJ, Russell AS, Skeith K. Lack of congruence in the ratings of patients' health status by patients and their physicians. Med Decis Making. 2001;21(2):113-121.

36. Mantyselka P, Kumpusalo E, Ahonen R, Takala J. Patients'versus general practitioners' assessments of pain intensity in primary care patients with non-cancer pain. Br J Gen Pract. 2001;51(473):995-997.

37. Petersen MA, Larsen H, Pedersen L, Sonne N, Groenvold M. Assessing health-related quality of life in palliative care: comparing patient and physician assessments. Eur J Cancer. 2006;42(8):1159-1166.

38. Yalcin I, Viktrup L. Comparison of physician and patient assessments of incontinence severity and improvement. Int Urogynecol J Pelvic Floor Dysfunct. 2007;18(11):1291-1295.

39. Srikrishna S, Robinson D, Cardozo L, Gonzalez J. Is there a discrepancy between patient and physician quality of life assessment? Neurourol Urodyn. 2009;28(3):179-182.

40. Leong KP, Chong EY, Kong KO, et al. Discordant assessment of lupus activity between patients and their physicians: the Singapore experience. Lupus. 2010;19(1):100-106.

41. Cappelleri JC, Bushmakin AG, Sadosky AB, Lionberger DR. Health status and patient-reported osteoarthritis severity in clinical practice. In: Functional Impairment. Hauppauge, NY:Nova Science Publishers. In Press.

Diabetes, Metabolic Syndrome and Obesity: Targets and Therapy

Dovepress

\section{Publish your work in this journal}

Diabetes, Metabolic Syndrome and Obesity: Targets and Therapy is an international, peer-reviewed open-access journal committed to the rapid publication of the latest laboratory and clinical findings in the fields of diabetes, metabolic syndrome and obesity research. Original research, review, case reports, hypothesis formation, expert opinion and commentaries are all considered for publication. The manuscript management system is completely online and includes a very quick and fair peer-review system, which is all easy to use. Visit http://www.dovepress.com/testimonials.php to read real quotes from published authors. 